Pacific Journal of Mathematics

STRONG LIE IDEALS 


\section{STRONG LIE IDEALS}

\section{ALBERT J. KARAM}

$R$ is 2-torsion free semiprime with $2 R=R$. A Lie ideal, $U$, of $R$-strong if $a u a \in U$ for all $a \in R, u \in U$. One shows that $U$ contains a nonzero two-sided ideal of $R$. If $R$ has an involution, $*$, (with skew-symmetric elements $K$ ) a Lie ideal, $U$, of $K$ is $K$-strong if $k u k \in U$ for all $k \in K, u \in U$. It is shown that if $R$ is simple with characteristic not 2 and either the center, $Z$, is zero or the dimension of $R$ over the center is greater than 4 , then $U=K$. If $R$ is a topological annihilator ring with continuous involution and if $U$ is closed $K$-strong Lie ideal, $U=C \cap K$ where $C$ is a closed two-sided ideal of $R$. A Lie ideal, $U$, of $K$ is $H K$-strong if $u^{3} \in U$ for all $u \in U$. A result similar to the above result for $K$-strong Lie ideals can be shown. Let $R$ be a simple ring with involution such that $Z=(0)$ or the dimension of $R$ over $Z$ is greater than 4 . Let $\phi$ be a nonzero additive map from $R$ into a ring $A$ such that the subring of $A$ generated by $\{\phi(x): x \in R\}$ is a noncommutative, 2-torsion free prime ring. Suppose $\phi\left(x y-y^{*} x^{*}\right)=\phi(x) \phi(y)-\phi\left(y^{*}\right) \phi\left(x^{*}\right)$ for all $x, y \in R$. As an application of the above theory, $\phi$ is shown to be an associative isomorphism.

1. Introduction. $R$ will denote a semiprime ring such that $2 R=R$ and if $2 r=0$, then $r=0$. We call the latter property 2torsion free. $Z$ will denote the center of $R$. If $R$ has an involution, *, defined on it, $S$ and $K$ will be the set of symmetric and skewsymmetric elements respectively. The Lie and Jordan products are $[x, y]=x y-y x$ and $x \circ y=x y+y x$ for any $x, y \in R$. If $X, Y \subseteq R$, $[X, Y]$ will denate the additive subgroup generated by the set $\{[x, y]: x \in X$ and $y \in Y\}$. An additive subgroup, $U$, of $R$ is a Lie ideal of $R$ if $[U, R] \subseteq U$. If $R$ has an involution, we can similarly define a Lie ideal of $K$.

This paper is concerned with the study of different classes of Lie ideals of both $R$ and $K$. A Lie ideal, $U$, of $R$ is said to be $R$-strong if $a u a \in U$ for all $a \in R, u \in U$. If $U$ is a Lie ideal of $K, U$ is $K-(H K-)$ strong if $k u k \in U\left(u^{3} \in U\right)$ for all $k \in K, u \in U$.

In the classical theory of the Lie structure of an associative ring, the main theorem [6; Th. 1.3] states: if $R$ is simple and $U$ is a Lie ideal of $R$, either $U \subseteq Z$ or $[R, R] \subseteq U$. We attempt to develop some criteria for differentiating between Lie ideals of $R$ containing $[R, R]$ and $R$ itself. Similar criteria are developed for Lie ideals of $K$. We 
will have occasion to use the following results of Herstein [6; pp $1,5,10$, and 28]:

(i) $R$ has no one-sided ideals which are nil of bounded index;

(ii) If $a \in R$ is such that $[\alpha,[\alpha, x]]=0$ for all $x \in R$, then $a \in Z$;

(iii) Let $R$ be simple with involution and characteristic not 2 . If $Z=(0)$ or the dimension of $R$ over $Z$ is greater than 4 , then $R=$ $\bar{S}=\bar{K}$ where $\bar{S}$ and $\bar{K}$ are the subrings of $R$ generated by $S$ and $K$ respectively.

If $X \subseteq R, \mathscr{R}(X)=\{a \in R: X a=(0)\}$ and $\mathscr{L}(X)=\{a \in R: a X=$ $(0)\}$. The next two lemmas are analogs of a results of Baxter [3; p. 2].

Lemma 1.1. If $U$ is a Lie ideal of $R$ such that $u^{2}=0$ for all $u \in U$, then $U=(0)$.

Proof. Let $u \in U, a \in R$. As $[u, a] \in U,[u, a]^{2}=0$. Therefore, $u a u a u=u[u, a]^{2}=0$ and $u R$ is nil of bounded index. By the previously mentioned results, $u R=(0)$. But $R$ is semiprime, so $\mathscr{L}(R)=(0)$. Thus $u=0$.

Lemma 1.2. Let $R$ have an involution, *. If $U$ is a Lie ideal of $K$ such that $u^{2}=0$ for all $u \in U$, then $U=(0)$.

Proof. Let $u, v \in U$, then $0=(u+v)^{2}-u^{2}-v^{2}=u v+v u$. As $[u, v] \in U, 2 u v \in U$. Since $2 R=R,[u v, K] \subseteq U$. Thus, for each $k \in K$, $u \circ[u v, k]=0$, and so, even more $v\{u \circ[u v, k]\}=0$. Since $u$ and $v$ anticommute, expansion of this expression yields $u v k u v=0$. Now suvs $\in K$ for any $s \in S$. So $u v(s u v s) u v=0$. Therefore, given $a \in R, a=s+k$ where $s \in S$ and $k \in K$, then $(u v) a(u v) a(u v)=0$. We conclude that $u v R$ is nil of bounded index. This guarantees $u v=0$ for all $u, v \in U$. Now, $-u k u=u[u, k]=0$. Repeating the previous arguments for $s \in$ $S$ and $k \in K$, we conclude that $u=0$.

2. $R$-strong Lie ideals. In this section $U$ will denote an $R$ strong Lie ideal. If $a, b \in R$ and $u, v \in U$, one can easily show that the following are in $U: a u b+b u a, a b u+u b a$, and $u a u$. We associate with $U$ the set $B_{U}=\{b \in R: a \circ b \in U$ for all $a \in R\}$. This set is a Lie ideal of $R$ and $u^{2} \in B_{U}$ for all $u \in U$. The latter can be seen by observing that if we set $b=u$ above, we obtain $a u^{2}+u^{2} a \in U$. Thus, via Lemma 1.1, $U \neq(0)$ implies $B_{U} \neq(0)$.

LEMMA 2.1.

(i) $B_{U}$ is an $R$-strong Lie ideal 
(ii) $u^{2} x u^{2} \in B_{U} \cap U$ for all $u \in U, x \in R$.

\section{Proof.}

(i) We know that $B_{U}$ is a Lie ideal of $R$. For arbitrary $x, y \in$ $R$ and $b \in B_{U},[x \circ b, y]$ and $[x, b] \circ y$ are in $U$. Thus, by adding and subtracting these terms, we have that $x b y-y b x$ and $b x y-y x b$ are in $U$. Now,

$$
\begin{aligned}
x(y b y)+(y b y) x= & \{(x y) b y-y b(x y)\} \\
& +\{y b(y x)-(y x) b y\}+\{y(b x+x b) y\} .
\end{aligned}
$$

Since each term on the right is in $U, x(y b y)+(y b y) x \in U$ and $B_{U}$ is $R$-strong.

(ii) As $u^{2} \in B_{U}, u^{2} x u^{2} \in B_{U}$. Moreover, $u^{2} x u^{2}=u(u x u) u \in U$. Therefore, $u^{2} x u^{2} \in B_{U} \cap U$.

THEOREM 2.2. $C=B_{U} \cap U$ is a nonzero two-sided ideal.

Proof. Note that $C$ is an $R$-strong Lie ideal. Also $C \neq(0)$ since if this were so, for each $u \in U, u^{2} R$ would be a nil right ideal of bounded index. Let $b \in C$ and $x, y \in R ; x b+b x \in U$. Also

$$
\begin{aligned}
(x b+b x) y+y(x b+b x)= & \{x(b y-y b)-(b y-y b) x\} \\
& +\{(y x) b+b(y x)\} \\
& +\{b(x y)+(y x) b\}
\end{aligned}
$$

As each term on the right is in $U,(x \circ b) \circ y \in U$. Thus, $x \circ b \in C$. Now $2 x b=x \circ b+[x, b] \in C$. Since $2 R=R, R b \subseteq C$. Similarly, $b R \cong C$. Thus $C$ is a nonzero two-sided ideal of $R$.

We note that $C$ is the same as the set $L_{U}=\{u \in U: u a \in U$ for all $a \in R\}$ which was used by Zuev [10] in his study of the Lie structure of $R$.

Corollary 2.3. If $R$ is simple and $U \neq(0), U=R$.

This corollary allows us to study the $R$-strong structure of the ring as it relates to minimal idempotents of $R$. If $e$ is a minimal idempotent, $e U e$ is an $e R e$-strong Lie ideal. Since $e R e$ is a division ring either $e U e=(0)$ or $e U e=e R e$. We use this fact to prove the next theorem.

THEOREM 2.4. Let $H$ be the homogeneous component of the socle which contains $e$. Then either $H \subseteq U$ or $H \cong \mathscr{L}(U) \cap \mathscr{R}(U)$. 
Proof. Recall that $H$ is a simple ring. The theorem then follows by considering $H \cap U$.

CoRollary 2.5. If $R$ is completely reducible, $U$ is the direct sum of the homogeneous components of the socle which it contains.

This result is similar to that of Kaplansky [7].

Assume that $R$ has the additional properties that $3 R=R$ and $R$ is 3-torsion free. Let $W$ be any Lie ideal of $R$ such that $u^{3} \in W$ for all $u \in W$. Let $u, v \in W$. We have $\alpha=2\left(v^{2} u+v u v+u v^{2}\right)=(u+v)^{3}+$ $(u-v)^{3}-2 u^{3} \in W, \beta=[v,[v, u]] \in W$ and $\gamma=\left[v^{2}, u\right] \in W$. From these we have: $3\left(v^{2} u+u v^{2}\right)=\alpha+\beta \in W, 6 v u v=\alpha-2 \beta \in W, 6 v^{2} u=\alpha+3 \gamma \in W$, and $6 u v^{2}=\alpha-3 \gamma \in W$. We now have enough to show a result similar to Theorem 2.2.

THEOREM 2.6. Let $W$ bo a Lie ideal of $R$ such that $u^{3} \in W$ for all $u \in W$. Then either $W$ contains a nonzero two-sided ideal or $u^{2} \in Z$ for all $u \in W$.

Proof. Let $a, b \in R$ and $u \in W$. Since $2 a[a, u]=[a,[a, u]]+\left[a^{2}, u\right] \epsilon$ $W$ and $2 R=R, a[a, u] \in W$. Linearization of this expression yields $a[b, u]+b[a, u] \in W$. Upon multiplication by 6 and replacement of $b$ by $v^{2}$, we obtain $6\left\{a\left[v^{2}, u\right]+v^{2}[a, u]\right\} \in W . \quad$ As $6 v^{2}[a, u] \in W, 6 a\left[v^{2}, u\right] \in W$ and this implies $a\left[v^{2}, u\right] \in W$. It immediately follows that $R\left[v^{2}, u\right] R \subseteq$ $W$ of $R\left[v^{2}, u\right] R \neq(0)$, we are finished.

Assume $R\left[v^{2}, u\right] R=(0)$ for all $u, v \in W$, then $\left[v^{2}, u\right] R$ is a nilpotent ideal, hence $\left[v^{2}, u\right]=0$ for all $u, v \in W$. As $\left[v^{2}, a\right]=[v, v a+a v] \in W$, $\left[v^{2},\left[v^{2}, a\right]\right]=0$. Thus, by remarks in $\S 1, v^{2} \in Z$.

The obvious corollary holds in the case where $R$ is simple.

3. $K$-strong Lie ideals, Let $R$ have an involution, *, and let $U$ be a $K$-strong Lie ideal. For $u, v \in U$ and $k, l \in K$, the following are in $U: k u l+l u k, k l u+u l k$, and $u k u$. We associate with $U$ the set $B(U)=\left\{b \in R: b a-a^{*} b^{*} \in U\right.$ for all $\left.a \in R\right\}$. This is the analog for Lie ideals of the set which Baxter [3] uses in his study of the Jordan structure of $S$. When there is no confusion, we write $B(U)=B$.

LEMMA 3.1.

(i) $B$ is a right ideal

(ii) $K B \cong B$

(iii) $u^{2} \in B$ for all $u \in U$ 
Proof. The proofs of (i) and (ii) are straightforward. We prove (iii). As $u \in U, u^{2} a-a^{*}\left(u^{2}\right)^{*}=u^{2} a-a^{*} u^{2}$. Then

$$
u^{2} a-a^{*} u^{2}=\left\{\left[u, u a+a^{*} u\right]\right\}+\left\{u\left(a-a^{*}\right) u\right\} .
$$

The first \{\} is in $U$ since $u a+a^{*} u \in K$. The second \{\} is in $U$ since $\left(a-a^{*}\right) \in K$ and $U$ is $K$-strong.

Now from Lemma 1.2, we know that if $U \neq(0), B \neq(0)$.

For $u \in U, k \in K, a \in R$ and $b, c \in B$, direct computation leads to the following facts: $a c^{*} b \in B, c^{*} b \in B, b k b^{*} \in B \cap U$, and $u k u \in B \cap U$.

THEOREM 3.2. Let $R$ be a simple ring with characteristic not 2. If $Z=(0)$ or the dimensinn of $R$ over $Z$ is greater than 4 , then $U=$ $K$.

The proof of this essentially the same as the proof of Theorem 7 [3; p. 7]. As a corollary, we include a slight extension of a theorem of Baxter [1; p. 74].

CoRollary 3.3. Let $R$ be as in the theorem. SoK, the additive subgroup of $R$ generated by the set $\{s \circ k: s \in S$ and $k \in K\}$ is a K-strong Lie ideal and hence $S \circ K=K$.

The following results on $\mathscr{L}(B)$ and $\mathscr{L}(U)$ will be particularly useful in the next section.

THEOREM 3.4. $\mathscr{L}(B)$ is a self-adjoint two-sided ideal.

Proof. The proof is similar to the proof of Theorem 2 [4; p. 563].

Knowing that $\mathscr{L}(B)$ is a two-sided ideal, we can easily show that $\mathscr{L}(B) \cap B=(0)$ and $\mathscr{L}(B) \cap U=(0)$.

\section{THEOREM 3.5. $\mathscr{L}(U \cap B)=\mathscr{L}(U)$.}

Proof. It suffices to show $\mathscr{L}(U \cap B) \subseteq \mathscr{L}(U)$. Let $b \in U \cap B$, $k \in K$, and $x \in \mathscr{L}(U \cap B)$. As $b k-k b \in U \cap B, x k b=-x(b k-k b)=0$. Thus, $\mathscr{L}(U \cap B) K \subseteq \mathscr{L}(U \cap B)$.

Let $u \in U$, then $u^{3} \in U \cap B$ so $x u^{3}=0$. Since $u^{2} k+k u^{2} \in U \cap B$, $x u^{2} k u=x\left(u^{2} k+k u^{2}\right) u=0$. Let $a \in R ; u a^{*}+a u \in K$, therefore $0=$ $x u^{2}\left(u a^{*}+a u\right) u=x u^{2} a u^{2}$. If we replace $a$ by $a x$, we have $\left(x u^{2} a\right)^{2}=0$. That is, $x u^{2} R$ is a nil ideal of bounded index and so $x u^{2}=0$ for any 
$u \in U$. Upon linearization we obtain

$$
x u v=-x v u \text { for } u, v \in U .
$$

Since $x u v u=-x v u^{2}=0$ and $v k v \in U$, we have

$$
x u(v k v) u=0 \text {. }
$$

Let $w \in U$ and $s \in S ; x u v(w s+s w) v u=0$. Replacement of $x$ by $x w$, expansion of the expression, and repeated use of (3.5.1) yields, $0=-x w v u s w v u$. By repeated use of (3.5.1) and finally (3.5.2), we have $x w v u k w v u=0$. Given $a \in R$, since $a=s+k$ for some $s \in S$ and $k \in K$, we can write $x w v u a w v u=0$. Replace $a$ by $a x$ to obtain

$$
x w v u(a x) w v u=0 .
$$

Then $x w v u R$ is a nilpotent ideal so $x w v u=0$. As $u k-k u \in U$.

$$
0=x w v(u k-k u)=-x w v k u \text {. }
$$

Let $s \in S ; x w v(w s+s w) v=0$. Moreover, since $x w v w s v=0$, we have $x w v s w v=0$. From (3.5.3), $x w v k w v=0$. As before, this implies

$$
x w v=0 \text {. }
$$

Immediately, $0=x w(v k-k v)=-x w k v$. In particular $x w k w=0$. Since $s w s \in K, x w(s w s) w=0$. Also, $0=x w(s w k-k w s) w=x w s w k w$. Again, letting $a=s+k$ for $a \in R$, we have $x w a w a w=0$. Via the same techniques, $x w=0$ or $x \in \mathscr{L}(U)$. Hence, $\mathscr{L}(U \cap B) \subseteq \mathscr{L}(U)$.

4. Topological annihilator rings. In this section $R$ will denote a semiprime topological annihilator ring with continuous involution such that $2 R=R$ and if $\left\{2 x_{\alpha}\right\}$ is a net convergent to $0 \in R$, then $\left\{x_{\alpha}\right\}$ is also a net convergent to 0 . $U$ will be a closed $K$-strong Lie ideal.

The definition of an annihilator ring says that $\mathscr{L}(R)=\mathscr{R}(R)=$ (0) and if $A(L)$ is a closed right (left) ideal not equal to $R$, then $\mathscr{L}(A) \neq(0) \mathscr{R}(L) \neq(0)$. So if $B=B(U), H=\mathscr{L}(B) \oplus B$ is dense in $R$. It is easy to show that if $U$ is closed, $B$ is closed. If $X \subseteq$ $R, C l(X)$ will denote to topolopical closure of $X$.

The following results have proofs which are similar to those given by Baxter in [3; p. 4].

\section{THEOREM 4.1.}

(i) $B$ is a two-sided ideal

(ii) $\{\mathscr{L}(B)\}^{*}=\mathscr{L}\left(B^{*}\right)$ 
(iii) $B=B^{*}$

(iv) $U \subseteq B$.

For any $x, y \in R$, we adopt the following notation: $(x, y)_{L}=$ $x y-y^{*} x^{*}$ and $(x, y)_{J}=x y+y^{*} x^{*}$. Using the results of the last theorem, we prove

TheOREM 4.2. $U=C \cap K$ where $C$ is a closed two-sided ideal.

Proof. Let $V$ be the additive subgroup of $S$ generated by the set $\left\{(u, a)_{J}: u \in U\right.$ and $\left.a \in R\right\}$. If we show $(U+V)$ to be a right ideal, since it is self-adjoint, it must be a two-sided ideal.

Since $U \subseteq B,(u, a)_{L}=u a+a^{*} u \in U$ for all $a \in R$. Let $c \in R$, then

$$
a u c+c^{*} u a^{*}=\left((a, u)_{L}, c\right)_{L}+\left(u,\left(-a^{*} c\right)\right)_{L} \in V
$$

and

$$
a u c-c^{*} u a^{*}=\left((a, u)_{L}, c\right)_{J}+\left(u,\left(-a^{*} c\right)\right)_{J} \in V .
$$

Since $2 R=R$, for any $2 d \in R, u(2 d)=(u, d)_{L}+(u, d)_{J} \in U+V$. Thus, $U R \subseteq U+V$. Also,

$$
\begin{aligned}
(u, a)_{J}(2 d)= & (u, a d)_{L}+\left\{a^{*} u(-d)+(-d)^{*} u a\right\}+(u, a d)_{J} \\
& +\left\{d^{*} u a-a^{*} u d\right\} \in U+V
\end{aligned}
$$

and $V R \subseteq U+V$. Thus $(U+V) R \leqq U+V$, or the desired conclusion that $(U+V)$ is a two-sided ideal.

Let $C=C l(U+V) . \quad U \subseteq C \cap K$. Let $x \in C \cap K$. There exists a net $\left\{u_{\alpha}+v_{\alpha}\right\}$ such that $u_{\alpha}+v_{\alpha} \rightarrow x$ where $u_{\alpha} \in U$ and $v_{\alpha} \in V$. As $x \in K,\left(u_{\alpha}+v_{\alpha}\right)^{*}=-u_{\alpha}+v_{\alpha} \rightarrow x^{*}=-x$. Thus $u_{\alpha}-v_{\alpha} \rightarrow x$. By subtracing these expressions we obtain $2 u_{\alpha} \rightarrow 2 x$. Therefore $u_{\alpha} \rightarrow x$. Since $u_{\alpha} \in U$ and $U$ is closed, $x \in U$. Hence, $C \cap K=U$.

5. $H K$-strong Lie ideals. In this section $U$ is an $H K$-strong Lie ideal. $R$ will have those properties as described in $\S 1$. We further assume that $3 R=R$ and $R$ is 3-torsion free. $H K$-strong Lie ideals were defined by Herstein [5]. Baxter [2; p. 393] showed that if $R$ is simple with either $Z=(0)$ or the dimension of $R$ over $Z$ greater than 16 with $U \nsubseteq Z$, then $U=K$. This can be refined by using entirely different techniques.

As before, we associate with $U$ the set $B(U) . \quad B$ is a right ideal and $K B \subseteq B$. However, we are no longer guaranteed that $u^{2} \in B$ for all $u \in U$. Hence the possibility that $B=(0)$ does arise.

Lemma 5.1. Let $u, v, w \in U$ and $k \in K$. 
(i) $6 v u v \in U$

(ii) $6(u v w+w v u) \in U$

(iii) $u v(w k-k w)+(w k-k w) v u \in U$

(iv) $u^{2} v-v u^{2} \in B$.

Proof. (i) and (ii) follow in a manner similar to the remarks preceding Theorem 2.6. (iii) holds because $2 R=R$ and $3 R=R$. Finally (iv) can be verified in the same manner as $[6 ; \mathrm{p} .33]$.

If $B=(0), u^{2} v-v u^{2}=0$ for all $u, v \in U$. Let $s \in S$. Since $\left[u^{2}, s\right]=$ $[u, u s+s u] \in U,\left[u^{2},\left[u^{2}, s\right]\right]=0$. Also, if $k \in K,\left[u^{2},[u, k]\right]=0$, therefore $\left[u^{2},\left[u^{2}, k\right]\right]=\left[u^{2}, u_{\circ}[u, k]\right]=0$. We know that this implies

$$
\left[u^{2},\left[u^{2}, a\right]\right]=0
$$

for all $a \in R$. Thus, from the first section, $u^{2} \in Z$.

We now refine Baxter's theorem.

THEOREM 5.2. Let $R$ be simple and of characteristic not 2 or 3 . If $Z=(0)$ or the dimension of $R$ over $Z$ is greater than 4, then either $U=K$ or $U^{2} \in Z$ for all $u \in U$.

Proof. If $B \neq(0)$, by the remarks preceding Lemmas 1.1 and 5.1 we have the alternative result.

We relate the notations of $K$ - and $H K$-strong Lie ideals by calling attention to the fact that if $U$ is $H K$-strong, $B \cap U$ is $K$-strong. Clearly $B \cap U$ is a Lie ideal. If $k \in K$ and $u \in B \cap U$, then $[k,[k, u]]=$ $k^{2} u+u k^{2}-2 k u k$. Now, $k^{2} u+u k^{2} \in B \cap U$ by the definition of $B$. Therefore, $k u k \in B \cap U$ since $2 R=R$.

Herstein [6; p. 28] has shown that $K^{2}$ is a Lie ideal of $R$. It is not difficult to show that if $U$ is an $H K$-strong Lie ideal such that $B \cap U=(0)$, then any $x \in B \cap S$ commutes with every element in $K^{2}$. We need this fact to prove

THEOREM 5.3. Let $R$ be a topological anninilator ring with properties as described in the previous section. Assume also that $3 R=R$ and if $\left\{3 x_{\alpha}\right\}$ is a net convergent to $0 \in R,\left\{x_{\alpha}\right\}$ is a net converging to 0. If $U$ is a closed $H K$-strong Lie ideal, then either $u^{2} \in Z$ for all $u \in U, U$ contains the intersection of $K$ with a closed two-sided ideal, or $u^{2} v-v u^{2} \in \mathscr{L}(K)$ for all $u, v \in U$.

Proof. If $B=(0), u^{2} \in Z$. Assume $B \neq(0)$ and $B \cap U \neq(0)$. 
Since $B \cap U$ is $K$-strong, Theorem 4.2 guarantees the existence of $C$, a closed two-sided ideal, such that $C \cap K=B \cap U \subseteq U$.

Let $B \cap U=(0)$. As $K^{2}$ is a Lie ideal of $R, t=u^{2} v-v u^{2} \in K^{2} \cap$ $(B \cap S)$. Also, by the remarks preceding the theorem, $[t,[t, a]]=0$ for all $a \in R$. Therefore, $t \in Z$. Let $k \in K ; t k+k t=t k-k^{*} t^{*} \in B \cap U=$ (0). Therefore, $t k=0$ or $t=u^{2} v-v u^{2} \in \mathscr{P}(K)$.

7. Application. We now parallel some of the results obtained by Small [9] and Riedlinger [8] concerning an additive mapping whose multiplicative property is defined relative to an involution. Let $R$ be a simple ring with involution, $*$, and characteristic not 2 such that $Z=(0)$ or the dimension of $R$ over $Z$ is greater than 4 . Notice that under these conditions $R$ cannot be commutative. Let $\phi$ be a nozero additive mapping from $R$ into an associative ring $A$. Assume $R^{\prime}=$ $\overline{\phi(R)}$, the subring of $A$ generated by $\{\phi(r): r \in R\}$, is a noncommutative prime ring such that $2 R^{\prime}=R^{\prime}$ and $R^{\prime}$ is 2-torsion free. Let $\phi$ enjoy the further property that $\phi\left(x y-y^{*} x^{*}\right)=\phi(x) \phi(y)-\phi\left(y^{*}\right) \dot{\phi}\left(x^{*}\right)$ for all $x, y \in R$. We would like to show that $\phi$ is an associative isomorphism. We will have occasion to use the following theorem by Baxter $[1 ; \mathrm{p}$. 73] which was slightly modified by Herstein [6; p. 29]: If $R$ is such that $2 R=R$ and $\bar{K}=R$, then $S=K \circ K$, the additive subgroup of $R$ generated by the set $\{k \circ l: k, l \in K\}$.

The next lemma is the key to much of what follows.

Lemma 6.1. $\operatorname{Ker} \phi \cap K=(0)$.

Proof. We show $\operatorname{Ker} \phi \cap K$ to be a $K$-strong Lie ideal. Let $l \in$ $\operatorname{Ker} \phi \cap K$ and $k \in K$. Since $\phi([k, l])=[\phi(k), \phi(l)]=0$, Ker $\phi \cap K$ is a Lie ideal of $K$. Thus $[k,[k, l]] \in \operatorname{Ker} \phi \cap K$ or $\phi([k,[k, l]])=(0)$. We may expand this and obtain

$$
\dot{\phi}([k,[k, l]])=\dot{\phi}\left(k^{2} l-2 k l k+l k^{2}\right)=\phi\left(k^{2} l+l k^{2}\right)-2 \phi(k l k)=0 .
$$

Now, $\dot{\phi}\left(k^{2} l+l k^{2}\right)=\dot{\phi}\left(k^{2}\right) \phi(l)+\phi(l) \dot{\phi}\left(k^{2}\right)=0$. Therefore $\phi(k l k)=0$ or $\operatorname{Ker} \phi \cap K$ is a $K$-strong Lie ideal.

By Theorem 3.2 either $\operatorname{Ker} \phi \cap K=(0)$ or $\operatorname{Ker} \dot{\phi} \cap K=K$. Assume the latter. For $s, t \in S$ and $k, l \in K,[\phi(k), \dot{\phi}(l)]=0$ and $[\dot{\phi}(k), \dot{\phi}(s)]=0$. As $[s, t] \in K, 0=\dot{\phi}([s, t])=[\dot{\phi}(s), \phi(t)]$. Because any $x \in R$ can be written as $x=s+k$, we have $[\phi(x), \phi(y)]=0$ for all $x, y \in R$. Therefore, $R^{\prime}$ is commutative, a contradiction. Thus $\operatorname{Ker} \phi \cap K=(0)$.

Let $x, y \in R$, then 


$$
\begin{aligned}
\phi\left(\left(x y-y^{*} x^{*}\right) x^{*}-x\left(x y-y^{*} x^{*}\right)^{*}\right)= & \left\{\phi(x) \phi(y)-\phi\left(y^{*}\right) \phi\left(x^{*}\right)\right\} \phi\left(x^{*}\right) \\
& -\phi(x)\left\{\phi\left(y^{*}\right) \phi\left(x^{*}\right)-\phi(x) \phi(y)\right\} .
\end{aligned}
$$

If $y=s$, we can write,

$$
\phi\left(\left(x y-y^{*} x^{*}\right) x^{*}-x\left(y^{*} x^{*}-x y\right)\right)=\phi\left(x^{2} s-s x^{*^{2}}\right)=\phi\left(x^{2}\right) \dot{\phi}(s)-\phi(s) \phi\left(x^{*^{2}}\right)
$$

and

$$
\begin{aligned}
& \left\{\phi(x) \phi(y)-\phi\left(y^{*}\right) \phi\left(x^{*}\right)\right\} \phi\left(x^{*}\right)-\phi(x)\left\{\phi\left(y^{*}\right) \phi\left(x^{*}\right)-\phi(x) \phi(y)\right\} \\
& =(\phi(x))^{2} \phi(s)-\phi(s)\left(\phi\left(x^{*}\right)\right)^{2}
\end{aligned}
$$

This can be rewritten as

$$
\left\{\phi\left(x^{2}\right)-(\phi(x))^{2}\right\} \phi(s)=\phi(s)\left\{\phi\left(x^{*^{2}}\right)-\left(\phi\left(x^{*}\right)\right)^{2}\right\}
$$

for all $x \in R$ and $s \in S$.

Lemma 6.2. For any $s \in S$ and

$$
k \in K,\left\{\phi\left(s^{2}\right)-(\phi(s))^{2}\right\} \quad \text { and } \quad\left\{\phi\left(k^{2}\right)-(\phi(k))^{2}\right\}
$$

are in $Z^{\prime}$, the center of $R^{\prime}$.

Proof. Set $u$ equal to either $\left\{\phi\left(s^{2}\right)-(\phi(s))^{2}\right\}$ or $\left\{\phi\left(k^{2}\right)-(\phi(k))^{2}\right\}$. From (6.1.1), $\phi(s) u=u \phi(s)$. Consider $2 \phi\left(t_{1} t_{2} \cdots t_{n}\right)$ where $t_{1} \in S$. We write

$$
\begin{aligned}
2 \phi\left(t_{1} t_{2} \cdots t_{n}\right)= & \phi\left(t_{1} t_{2} \cdots t_{n}+t_{n} \cdots t_{2} t_{1}\right) \\
& +\phi\left(t_{1} t_{2} \cdots t_{n}-t_{n} \cdots t_{2} t_{1}\right) \\
= & \phi\left(t_{1} t_{2} \cdots t_{n}+t_{n} \cdots t_{2} t_{1}\right) \\
& +\left\{\phi\left(t_{1}\right) \phi\left(t_{2} \cdots t_{n}\right)-\phi\left(t_{n} \cdots t_{2}\right) \phi\left(t_{1}\right)\right\} .
\end{aligned}
$$

By induction, $u$ commutes with $\phi\left(t_{2} \cdots t_{n}\right)$ and $\phi\left(t_{n} \cdots t_{2}\right)$. Since $t_{1} t_{2} \cdots t_{n}+t_{n} \cdots t_{2} t, \in S, u$ commutes with $\phi\left(t_{1} t_{2} \cdots t_{n}+t_{n} \cdots t_{2} t_{1}\right)$. Thus, $\left[u, \phi\left(t_{1} t_{2} \cdots t_{n}\right)\right]=0$. That is, $u$ commutes with $\phi(\bar{S})$. But under our hypothesis, $\bar{S}=R$. Hence, $u$ commutes with $\phi(R)$ and, indeed, with $\overline{\phi(R)}=R^{\prime}$. Thus $u \in Z^{\prime}$.

COROLLARY 6.3.

$$
\left\{\phi\left(x^{2}\right)-(\phi(x))^{2}\right\} \in Z^{\prime} \quad \text { for all } x \in R .
$$

Proof. If $x=s+k$, since $\phi(s k+k s)-\{\phi(s) \phi(k)+\phi(k) \phi(s)\}=0$, $\left\{\phi\left(x^{2}\right)-(\phi(x))^{2}\right\}=\left\{\phi\left(s^{2}\right)-(\phi(s))^{2}\right\}+\left\{\phi\left(k^{2}\right)-(\phi(k))^{2}\right\} \in Z^{\prime}$.

Let $x, y \in R$. If we linearize (6.3.1), we obtain 


$$
\phi(x y+y x)-\{\phi(x) \phi(y)+\phi(y) \phi(x)\} \in Z^{\prime} .
$$

In particular, for $s, t \in S, \phi(s t+t s)-\{\phi(s) \phi(t)+\phi(t) \phi(s)\} \in Z^{\prime}$. Also, $\phi(s t-t s)-\{\phi(s) \phi(t)-\phi(t) \phi(s)\}=0$. Addition of these terms leads us to $\phi(s t)-\phi(s) \phi(t) \in Z^{\prime}$. Similarly, we can show that $\phi(k l)-\phi(k) \phi(l) \in$ $Z^{\prime}$ for $k, l \in K$.

For notational convenience, let $\phi(x y)-\phi(x) \phi(y)=x^{y}$ for any $x, y \in$ $R$. Thus the above says that $s^{t}, k^{l} \in Z^{\prime}$. The definition of $\phi$ tells us that $s^{k}=-k^{s}$. Also, we have $k^{l}=l^{k}$. Since these terms are in $Z^{\prime}$, $\phi(s) k^{l}-l^{k} \phi(s)=0$. Upon expansion and rearrangement of terms, we obtain

$$
\{\phi(s k l-l k s)\}-\{\phi(s) \phi(k) \phi(l)-\phi(l) \phi(k) \phi(s)\}=0 .
$$

We can write $\phi(s k-k s)=\phi(s k) \phi(l)-\phi(l) \phi(k s)$. Replacement of this in (6.4.1) and rearrangement of terms yields

$$
s^{k} \phi(l)-\phi(l) k^{s}=0
$$

or

$$
s^{k} \phi(l)=\phi(l) k^{s}=-\phi(l) s^{k} .
$$

Let $m \in K$, by the above, there exists $z^{\prime} \in Z^{\prime}$ such that $\phi(m l+l m)=$ $\phi(m) \phi(l)+\phi(l) \phi(m)+z^{\prime}$. As a result of (6.4.2) and this relation we have that $s^{k} \phi(m l+l m)=\phi(m l+l m) s^{k}$ or $s^{k}$ commutes with $\phi(K \circ K)$. The preliminary remarks guarantee for us that $K \circ K=S$. So, using an argument exactly like that in Lemma 6.2, we can show

$$
s^{k} \in Z^{\prime} \text {. }
$$

Lemma 6.4. $x^{y} \in Z^{\prime}$ for all $x, y \in R$.

The proof follows directly from (6.4.3) and the remarks immediately after Corollary 6.3.

CoRollary 6.5. If $Z^{\prime}=(0), \phi$ is an associative isomorphism.

Proof. As $Z^{\prime}=(0), \phi(x y)-\phi(x) \phi(y)=0$. Thus $\phi$ is an associative homomorphism and $\overline{\phi(R)}=\phi(R)$. Moreover, since $R$ is simple, $\phi$ is an associative isomorphism.

Let $z^{\prime}(\neq 0) \in Z^{\prime}$. Since $\mathscr{A}\left(z^{\prime}\right)=\left\{r^{\prime} \in R^{\prime}: r^{\prime} z^{\prime}=0\right\}$ is a two-sided ideal in a prime ring, $\mathscr{A}\left(z^{\prime}\right)=(0)$.

LEMma 6.6. $k^{s}=s^{k}=0$ for all $s \in S, k \in K$.

Proof. From (6.4.2) $s^{k} \phi(l)=-\phi(l) s^{k}$ for $l \in K$. By Lemma 6.4, $s^{k} \in$ 
$Z^{\prime}$, therefore $s^{k} \phi(l)=0$. Suppose $s^{k} \neq 0$. By the remarks preceding the lemma, we have $\phi(l)=0$, that is, $K \cong \operatorname{Ker} \phi$. Therefore, Ker $\phi \cap$ $K=K$, a contradiction. We conclude that $0=s^{k}=-k^{s}$.

CoRollary 6.7. $\dot{\phi}(x y-y x)=\dot{\phi}(x) \dot{\phi}(y)-\dot{\phi}(y) \dot{\phi}(x)$ for $x, y \in R$.

We have shown that when $Z^{\prime}=(0)$, then $\phi$ is an associative isomorphism. Therefore, the following theorem is proved except when $Z^{\prime} \neq(0)$.

\section{THEOREM 6.8. $\phi$ is an associative isomorphism.}

Proof. From Lemma 6.6, $\left(s^{2}\right)^{k}-\phi(s) s^{k}=0$. Expansion and rearrangement of terms leads to $\left(s^{2}\right)^{k}-\phi(s) s^{k}=(s)^{s k}-s^{s} \dot{\phi}(k)=0$. From Lemma 6.4, $(s)^{s k} \in Z^{\prime}$ so $s^{s} \phi(k) \in Z^{\prime}$. Let $l \in K$. There exist $z_{1}^{\prime}$ and $z_{2}^{\prime}$ in $Z^{\prime}$ such that $s^{s} \phi(k)=z_{1}^{\prime}$ and $s^{s} \phi(l)=z_{2}^{\prime}$. As $s^{s} \in Z^{\prime}$, we can write $0=\left[z_{1}^{\prime}, z_{2}^{\prime}\right]=\left(s^{s}\right)^{2}[\phi(k), \phi(l)]$ for all $s \in S$ and $k, l \in K$.

If $\left(s^{s}\right)^{2} \neq 0$ for some $s \in S$, then by the remarks preceding Lemma 6.6, $[\phi(k), \phi(l)]=0$ for all $k, l \in K$. As $\phi([k, l])=[\phi(k), \phi(l)]=0$, we conclude that $[K, K] \subseteq \operatorname{Ker} \phi \cap K=(0)$. This implies $\bar{K}=R$ is commutative, a contradiction. So $\left(s^{s}\right)^{2}=0$ for all $s \in S$. Since the center of a prime ring is an integral domain, $s^{s}=0$. Upon linearization of this expression, we obtain $\phi(s t+t s)-\{\dot{\phi}(s) \phi(t)+\phi(t) \phi(s)\}=0$ for all $t, s \in S$.

For $k, l \in K, k^{l} \in Z^{\prime}$. Thus there exists $z_{3}^{\prime} \in Z^{\prime}$ such that $k^{l}-z_{3}^{\prime}=$ 0 . Since $k^{2} \in S,\left(k^{2}\right)^{l}=0$ and so $\left(k^{2}\right)^{l}-\phi(k)\left\{k^{l}-z_{3}^{\prime}\right\}=0$. Expansion and rearrangement of terms leads to $k^{k l}-k^{k} \phi(l)+z_{3}^{\prime} \dot{\phi}(k)=0$. In view of Lemma 6.4, there is an element $z_{4}^{\prime} \in Z^{\prime}$ such that $k^{k l}=z_{4}^{\prime}$. Therefore we can always find $z_{3}^{\prime}, z_{4}^{\prime}, \in Z^{\prime}$ such that $k^{k} \phi(l)=z_{3}^{\prime} \phi(k)+z_{4}^{\prime}$ where $k$ is an arbitrary fixed element in $K$ and $l$ is allowed to vary in $K$. Note that $k^{k} \in Z^{\prime}$. For $m \in K$, there are $z_{5}^{\prime}$ and $z_{6}^{\prime}$ in $Z^{\prime}$ such that $k^{k} \dot{\phi}(m)=z_{5}^{\prime} \phi(k)+z_{6}^{\prime}$. Thus $0=\left(k^{k}\right)^{2}[\dot{\phi}(l), \dot{\phi}(m)]=\left[k^{k} \phi(l), k^{k} \dot{\phi}(m)\right] . \quad$ Via the same argument as above, we can show $k^{k}=0$. Linearization of this expression leads to $\phi(k l+l k)-\{\phi(k) \phi(l)+\phi(l) \phi(k)\}=0$. Now, using this fact and the fact that both $\dot{\phi}(s k)-\phi(s) \dot{\phi}(k)=0$ and $\dot{\phi}(s t+t s)-\{\dot{\phi}(s) \dot{\phi}(t)+\dot{\phi}(t) \dot{\phi}(s)\}=0$, we have that

$$
\dot{\phi}(x y+y x)=\dot{\phi}(x) \dot{\phi}(y)+\dot{\phi}(y) \dot{\phi}(x)
$$

for all $x, y \in R$. From Corollary 6.7, we know

$$
\phi(x y-y x)=\phi(x) \phi(y)-\phi(y) \phi(x) .
$$

Addition of these two expressions yields $\phi(x y)=\phi(x) \dot{\phi}(y)$ or that $\dot{\phi}$ is an associative homomorphism. Therefore, $\overline{\phi(R)}=\phi(R)$ and $\operatorname{Ker} \phi=(0)$ 
since $R$ is simple. Hence $\dot{\phi}$ is an associative isomorphism.

\section{REFERENCES}

1. W. E. Baxter, Lie simplicity of a special class of associative rings II, Trans. Amer. Math. Soc., 87 (1958), 63-75.

2. — Concerning strong Lie ideal, Proc. Amer. Math. Soc., 11 (1960), 393-395.

3. - On rings with proper involution, Pacific J. Math., 27 (1968), 1-12.

4. - Topological rings with property (Y), Pacific J. Math., 30 (1969), 563-571.

5. I. N. Herstein, Lie and Jordan systems in simple rings with involution, Amer. J. Math., 78 (1956), 629-649.

6. I. N. Herstein, Topics in Ring Theory, Univ. of Chicago, Chicago, III. 1965: rev. ed. 1969 .

7. I. Kaplansky, Semi-automorphisms of rings, Duke Math. J., 14 (1947), 521-527.

8. R. F. Riedlinger, Mappings on Rings with Involution, Ph. D. Dissertation, University of Delaware, Newark, Delaware 1971.

9. L. B. Small, Mappings on Simple Rings with Involution, Ph. D. Dissertation, Yale University, New Haven, Conn., 1968.

10. I. Zuev, Lie ideal of an associative ring, Uspehi Mat. Nauk, 18 (1963), no. 1 (109), $155-158$.

Received July 26, 1971. The author wishes to thank Professor Willard E. Baxter for his invaluable help. This research was supported in part by NSF Grant GP-9611. This work presented here comprises part of the author's Ph. D. dissertation.

Universidad De los Andes, Mérida, Venezuela

AND

UNIVERSITY OF DELAWARE 



\section{PACIFIC JOURNAL OF MATHEMATICS}

\section{EDITORS}

\section{H. SAMElson}

Stanford University

Stanford, California 94305

C. R. Новву

University of Washington

Seattle, Washington 98105
J. DugunduI

Department of Mathematics University of Southern California

Los Angeles, California 90007

RICHARD ARENS

University of California

Los Angeles, California 90024

\section{ASSOCIATE EDITORS}
E. F. BECKENBACH
B. H. NeUmanN
F. WOLF
K. YosHIDA

\section{SUPPORTING INSTITUTIONS}

UNIVERSITY OF BRITISH COLUMBIA

UNIVERSITY OF SOUTHERN CALIFORNIA

CALIFORNIA INSTITUTE OF TECHNOLOGY

STANFORD UNIVERSITY

UNIVERSITY OF CALIFORNIA

UNIVERSITY OF TOKYO

MONTANA STATE UNIVERSITY

UNIVERSITY OF UTAH

UNIVERSITY OF NEVADA

WASHINGTON STATE UNIVERSITY

NEW MEXICO STATE UNIVERSITY

OREGON STATE UNIVERSITY

UNIVERSITY OF OREGON

OSAKA UNIVERSITY

UNIVERSITY OF WASHINGTON

* *

AMERICAN MATHEMATICAL SOCIETY

NAVAL WEAPONS CENTER 


\section{Pacific Journal of Mathematics}

\section{Vol. 43, No. $1 \quad$ March, 1972}

Alexander (Smbat) Abian, The use of mitotic ordinals in cardinal

arithmetic ....................................... 1

Helen Elizabeth. Adams, Filtrations and valuations on rings ......... 7

Benno Artmann, Geometric aspects of primary lattices .............. 15

Marilyn Breen, Determining a polytope by Radon partitions ........... 27

David S. Browder, Derived algebras in $L_{1}$ of a compact group .......... 39

Aiden A. Bruen, Unimbeddable nets of small deficiency .............. 51

Michael Howard Clapp and Raymond Frank Dickman, Unicoherent

compactifications ............................... 55

Heron S. Collins and Robert A. Fontenot, Approximate identities and the strict topology ................................... 63

R. J. Gazik, Convergence in spaces of subsets................. 81

Joan Geramita, Automorphisms on cylindrical semigroups ........... 93

Kenneth R. Goodearl, Distributing tensor product over direct product ..... 107

Julien O. Hennefeld, The non-conjugacy of certain algebras of

operators ................................... 111

C. Ward Henson, The nonstandard hulls of a uniform space ........... 115

M. Jeanette Huebener, Complementation in the lattice of regular

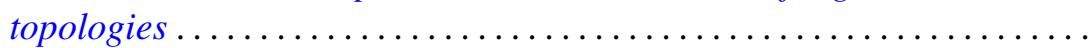

Dennis Lee Johnson, The diophantine problem $Y^{2}-X^{3}=A$ in a

polynomial ring .................................... 151

Albert Joseph Karam, Strong Lie ideals . . . . . . . . . . . . . . . . . . . . 157

Soon-Kyu Kim, On low dimensional minimal sets ............... 171

Thomas Latimer Kriete, III and Marvin Rosenblum, A Phragmén-Lindelöf

theorem with applications to $M(u, v)$ functions ..... . .

William A. Lampe, Notes on related structures of a universal algebra . . . . 189

Theodore Windle Palmer, The reducing ideal is a radical .

207

Kulumani M. Rangaswamy and N. Vanaja, Quasi projectives in abelian and module categories ................................ 221

Ghulam M. Shah, On the univalence of some analytic functions ......... 239

Joseph Earl Valentine and Stanley G. Wayment, Criteria for Banach

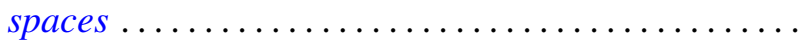

Jerry Eugene Vaughan, Linearly stratifiable spaces ............... 253

Zbigniew Zielezny, On spaces of distributions strongly regular with respect to partial differential operators ..................... 